\title{
PEG-coated patch parenchymal closure technique and initial outcomes during minimally invasive partial nephrectomy
}

\author{
Ryan McLarty, MD; Benjamin Beech, MD; Jan K. Rudzinski, MD; Blair St. Martin, MD; Howard Evans, MD
}

Division of Urology, Department of Surgery, University of Alberta, Edmonton, AB, Canada

Cite as: McLarty R, Beech B, Rudzinski JK, et al. PEG-coated patch parenchymal closure technique and initial outcomes during minimally invasive partial nephrectomy. Can Urol Assoc J 2022;16 (1):E44-7. http://dx.doi.org/10.5489/cuaj.7286

Published online August 26, 2021

\section{Abstract}

Introduction: The Hemopatch ${ }^{\circledR}$ is a novel polyethylene glycol-coated (PEG-coated) collagen patch that acts as a topical hemostatic agent. It has been applied to a variety of surgical techniques. Here, we present our series and technique using a PEG-coated patch for minimally invasive partial nephrectomy (MIPN).

Methods: We conducted a retrospective chart review of all patients undergoing MIPN by a single surgeon at a tertiary Canadian medical center between July and December 2018. We included patients if a PEG-coated patch was used to close the renal parenchymal defect. We also describe in detail our technique for laparoscopic patch deployment.

Results: A total of 17 patients met inclusion criteria, of whom 12 were male. Mean age was 63 years old. Median size of renal mass was $2.85 \mathrm{~cm}$ in largest dimension and median RENAL score was 6 . Hilar clamping was carried out in 12 (70.9\%) cases, with an average warm ischemic time of 16.1 minutes. The remainder of cases were performed off-clamp. Median estimated blood loss was 238 $\mathrm{mL}$ and the mean change in hemoglobin on postoperative day 1 compared to preoperatively was $21.2 \mathrm{~g} / \mathrm{L}$. The average length of stay was 1.76 days. No patient required blood transfusion or underwent angioembolization or re-operation for bleeding within 90 days. There were no Clavien-Dindo grade 3 or greater complications. Conclusions: A PEG-coated patch can be used safely and effectively in lieu of traditional two-layer renorrhaphy with acceptable outcomes. Larger, prospective series are required to ascertain its true value and cost-effectiveness.

\section{Introduction}

Partial nephrectomy is a first-line option for the surgical treatment of small renal masses. ${ }^{1}$ Partial nephrectomy rates have been increasing, with minimally invasive laparoscopic and robotic techniques being applied to more complex dissec- tions. $^{2}$ Partial nephrectomy is associated with a higher risk of postoperative complications compared to radical nephrectomy, especially when considering intraoperative blood loss and postoperative bleeding risk. ${ }^{3}$ The need for meticulous hemostasis is often balanced with needing to minimize ischemic time. While there is significant variability in the management of the renal parenchymal defect with respect to hemostasis, the main options include a combination of suture closure and hemostatic agents. ${ }^{4}$ Topical hemostatic agents have become commonplace in minimally invasive partial nephrectomy (MIPN) to reduce bleeding associated with the defect. ${ }^{4,5}$ There has been increasing interest in the use of a new synthetic hemostatic agent, the polyethylene glycol-coated (PEG-coated) collagen patch (Hemoaptch ${ }^{\circledR}$, Baxter healthcare Corporation) to control intraoperative bleeding. ${ }^{6}$ This solid patch can be precisely placed over the area of bleeding directly from the package without preparation, unlike other topical agents. Hemostasis is achieved through rapid tissue adherence facilitated by the PEG-coating and stimulated platelet activation and adhesion though its collagen layer. The use of this PEG-coated patch for hemostasis has been described across a number of surgical disciplines for both open and laparoscopic procedures. ${ }^{7,8}$ One area of concern in laparoscopy is unintentionally activating the PEG layer on adjacent tissue or blood while positioning the patch, which may complicate the ease of use of this patch in laparoscopy compared to open surgery. ${ }^{9}$

Herein, we describe our initial experience with using a PEG-coated patch in lieu of traditional renorrhaphy during MIPN. Furthermore, we describe our novel laparoscopic deployment technique, which facilitates quick patch deployment with minimal unintended tissue contact.

\section{Methods}

A retrospective chart review was performed of consecutive patients undergoing robotic-assisted MIPN by a single surgeon at the University of Alberta between July and December 2018. This quality assurance assessment met criteria for operational improvement activities and was exempt from 
local ethics review. During this time frame, a PEG-coated patch was used during the renorraphy, as discussed below in surgical technique. RENAL score was measured as per the method of Kutikov et al. ${ }^{10}$ The primary outcome of interest was postoperative angioembolization or surgical re-operation within 90 days. Secondary outcomes included 90-day Clavien-Dindo complications, estimated blood loss, blood transfusion requirements, postoperative hemoglobin change, length of hospital stay, and 90-day emergency department visit or hospital admission. Descriptive statistics were provided for the case series.

\section{Surgical technique}

All patients underwent robotic-assisted MIPN by a single surgeon. Two assistant ports were used to ensure both adequate suction and the ability to place pressure or pass equipment as needed. The method of hilar clamping was up to the discretion of the surgeon. Mass excision was performed using scissors, followed by a single running barbed sutured at the base of the defect anchored with clips. In lieu of a second layer closure aimed at re-approximating the renal cortex, a PEG-coated patch was used. Fig. 1 depicts preparation and deployment of the PEG-coated patch (Hemopatch ${ }^{\circledR}$, Baxter Healthcare Corporation). The PEG-coated patch $(27 \mathrm{~mm} \times$ $27 \mathrm{~mm}$, cost \$70 CAD, or $45 \mathrm{~mm} \times 45 \mathrm{~mm}$, cost \$185 CAD) was rolled and placed in the cut finger of a surgical glove with the blue-dotted side facing outwards (cost data from Baxter healthcare Corporation, Canada). The patch, protected by the latex glove cover, was introduced by the assistant

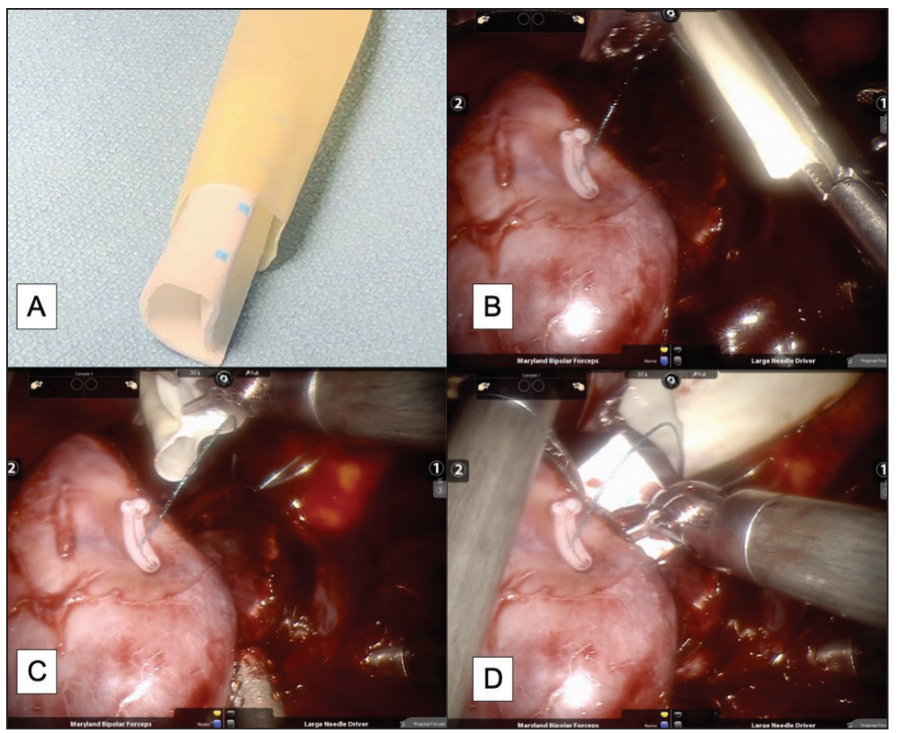

Fig. 1. (A) Preparation and deployment of a PEG-coated patch into a renal mass defect. The patch is rolled with the adhesive side facing inward and placed into the cut 5 th finger of a sterile glove. (B) The assistant deploys the glove finger into the field using a laparoscopic grasper. $(\boldsymbol{C}, \boldsymbol{D})$ The surgeon can manipulate the hemopatch onto the defect without contact with surrounding tisues and fluids. just beyond the renal parenchymal defect. The patch was then removed from the glove cover, allowing it to open PEG-coated side down onto the defect. Gentle, continuous pressure was placed over the patch for two minutes. If applicable, the hilum was unclamped and the procedure continued as per usual.

\section{Results}

Seventeen patients underwent robotic-assisted MIPN during the trial period, with all patients receiving a PEG-coated patch renorraphy. Complete followup of 90 days was available for all patients. Table 1 describes the characteristics of the sample. The mean age was 63.1 years and $70.6 \%$ were male. No patients were on anticoagulation therapy or antiplatelet therapy. Mean tumor size was $2.85 \mathrm{~cm}$ and mean numerical RENAL score was 6 (range 4-10). Most (70.6\%) patients had partial nephrectomy performed with hilar clamping compared to no-clamp, with a mean warm ischemic time of 16.1 minutes. No patients required additional hemostatic agents or sutures after placement of the PEG-coated patch. The mean estimated blood loss was $238 \mathrm{~mL}$, with a mean hemoglobin decline of $21.2 \mathrm{~g} / \mathrm{L}$ on postoperative day 1 . No patient required intraoperative or postoperative blood transfusion, re-operation, or angioembolization. There were no complications greater than Clavien-Dindo grade 3 and no patient experienced a 90-day emergency department visit or hospitalization. One patient developed postoperative urinary retention, and another developed a urinary tract infection treated with antibiotics.

\section{Discussion}

We demonstrated a novel technique for placement of a PEGcoated patched during MIPN. There is minimal experience with PEG-coated patches during MIPN. Only a single German series has described the feasibility and outcomes of a PEGcoated patch for hemostasis during MIPN. Imkamp et al first described the successful use of a PEG-coated patch in 2015 for seven patients undergoing zero-ischemia partial nephrectomy with no complications. ${ }^{11}$ This series of partial nephrectomy patients, of which $90 \%$ were laparoscopic, was subsequently updated in 2020 with 45 patients over a five-year period. ${ }^{12}$ In this update, they confirmed a PEG-coated patch can be applied to more complex partial nephrectomy patients but reported a $6 \%$ rate of intervention for postoperative bleeding.

Furthermore, we found that the use of a PEG-coated patch was feasible and safe, with minimal warm ischemic time. While two minutes of pressure is recommended for use of the patch, this time is made up with lack of formal renorrhaphy suturing and was not a concern in our experience. There are similarities and differences between this series and the previously reported series of PEG-coated patch use in 


\begin{tabular}{|c|c|}
\hline Patient variable $(n=17)$ & Outcome* \\
\hline Age (years) & $63.1(12.1)$ \\
\hline Male & $12(70.6 \%)$ \\
\hline Largest tumor dimension $(\mathrm{cm})$ & $2.85(0.87)$ \\
\hline RENAL score ${ }^{* *}$ & $6(4-10)$ \\
\hline POD1 hemoglobin drop (g/L) & $21.2(8.8)$ \\
\hline $\begin{array}{l}\text { No. with hilar clamp } \\
\text { Warm ischemic time (min) }\end{array}$ & $\begin{array}{c}12(70.6 \%) \\
16.1(2.4)\end{array}$ \\
\hline Estimated blood loss (mL) & $238(115)$ \\
\hline Blood transfusion & $0(0 \%)$ \\
\hline 90-day embolization or reoperation & $0(0 \%)$ \\
\hline 90-day emergency department visit/hospitalization & $0(0 \%)$ \\
\hline Length of stay (days) & $1.8(0.7)$ \\
\hline
\end{tabular}

* Reported as mean (standard deviation) for continuous variables and frequency (percentage) for categorical variables. ${ }^{*}$ Reported as median and range; excluded anterior/ posterior/neither designation.

partial nephrectomy. ${ }^{12}$ Surgical approach, technique with a hemostatic running suture in the defect base, and RENAL scores were similar. We did not use additional hemostatic agents and did not report any blood transfusion or intervention for bleeding. We did have a lower proportion of patients who received an off-clamp technique $(55.6 \%$ vs. $29.4 \%$ ). It is difficult to speculate reasons for different outcomes, considering our limited number of patients, but we did demonstrate acceptable safety in our limited series, with favourable warm ischemic times.

Minimal evidence exists regarding hemostasis during MIPN. The Canadian update of surgical procedures (CUSP) urology group recently developed a consensus describing best practices for managing bleeding during renorrhaphy: using a barbed absorbable hemostatic running stich, considering the use of gelatin thrombin agents, placing clips as opposed to knot tying sutures, and early unclamping to identify and ligate bleeding vessels. ${ }^{4}$ The use or consideration of PEG-coated patches was not endorsed by the consensus group, likely due to the minimal evidence for this technique.

There are several limitations with our series, namely the single-center, retrospective design of a limited number of patients. Most tumors had low RENAL scores and it is unknown if similar efficacy would be seen in more complex renal masses. Furthermore, none of the patients were on antiplatelet or anticoagulation and we cannot draw any conclusions in this group. We are also unable to comment on the efficacy of just using a PEG-coated patch without an initial barbed hemostatic stich.

There is currently no comparison between a PEG-coated patch and traditional renorrhaphy techniques with other hemostatic agents. This limits our ability to make conclusions regarding practicality, true efficacy, and cost-effectiveness. There are reports of a partial nephrectomy technique involving a fibrin sealant patch $\left(\operatorname{TachoSi}^{\circledR}\right)$. Antonelli et al found that there was no difference in postoperative complications between patients undergoing partial nephrectomy with either a fibrin sealant patch, Floseal $^{\circledR}$, or no hemostatic agent. ${ }^{13}$ Shigeta et al reported that there was a lower incident of pseudoaneurysm when using a fibrin sealant patch $\left(\right.$ Tachsea $\left.^{\circledR}\right)$ compared to standard of care. ${ }^{14}$

The role of PEG-coated patches in reducing pseudoaneurysm formation is unknown. A PEG-coated patch may have unique indications for expedited hemostasis during difficultto-control bleeding or to expedite prolonged clamp times. While feasible, further prospective studies are required to determine the utility of PEG-coated patches compared to tradition renorrhaphy techniques with other hemostatic agents.

\section{Conclusions}

A PEG-coated patch is a feasible and safe option in lieu of traditional renorrhaphy for MIPN. We also describe a laparoscopic deployment technique that could be adaptable to any minimally invasive procedure. Further study is required to inform us about the utility of a PEG-coated patch compared to traditional methods of renorrhaphy and other hemostatic agents.

Competing interests: The authors do not report any competing personal or financial interests related to this work.

This paper has been peer-reviewed.

\section{References}

1. Wilcox Vanden Berg RN, Basourakos SP, LaRussa S, et al. Management of the small renal mass: A 2020 update. Curr Oncol Rep 2020;22:69. https://doi.org/10.1007/s1 1912-020-00924-9

2. Alameddine M, Koru-Sengul T, Moore KJ, et al. Trends in utilization of robotic and open partial nephrectomy for management of cTl renal masses. Eur Urol Focus 2019;5:482-7. https://doi.org/10.1016/i. euf.2017.12.006

3. Mir MC, Derweesh I, Porpiglia F, et al. Partial nephrectomy vs. radical nephrectomy for clinical TI b and T2 renal tumors: A systematic review and meta-analysis of comparative studies. Eur Urol 2017;71:606-17. hitps://doi.org/10.1016/i.eururo.2016.08.060

4. Cheung DC, Wallis CJD, Possee S, et al. Canadian Update on Surgical Procedures (CUSP) Urology Group consensus for intraoperative hemostasis during minimally invasive partial nephrectomy. Can Urol Assoc J 2020;14:E387-93. https://doi.org/10.5489/cuaj.6579

5. Breda A, Stepanian SV, Lam JS, et al. Use of haemostatic agents and glues during laparoscopic partial nephrectomy: A multi-institutional survey from the United States and Europe of 1347 cases. Eur Urol 2007;52:798-803. htrps://doi.org/10.1016/i.eururo.2007.02.036

6. Lewis KM, Kuntze CE and Gulle H. Control of bleeding in surgical procedures: Critical appraisal of HEMOPATCH (Sealing Hemostat). Med Devices Evid Res 2015;9:1-10. https://doi.org/10.2147/ MDER.S90591

7. Lewis KM, Ikeme S, Olubunmi I, et al. Clinical effectiveness and versatility of a sealing hemostatic patch (HEMOPATCH) in multiple surgical specialties. Expert Rev Med Devices 2018;15:367-76. https://doi. org/10.1080/17434440.2018.1464909

8. Kapoor A, Wong ECL, Vasisth $G$, et al. A prospective, randomized, pilot trial of a polyethylene glycol (PEG)-coated collagen patch (Hemopatch $\left.{ }^{\circledR}\right)$ for intraoperative hemostasis during deceased donor renal transplant. Can Urol Assoc J 2020;14:E1-5. https://doi.org/10.5489/cuaj.5938 
9. Di Cesare T, Scarinci A, Cavaniglia D, et al. Efficacy of Hemopatch ${ }^{\circledR}$ in reducing postoperative bleeding after laparoscopic cholecystectomy: prospective and multicenter study. Integr Clin Med 2018;2:1-3. hitps:// doi.org/10.15761/ICM.1000133

10. (10) Kutikov A and Uzzo RG. The R.E.N.A.L. Nephrometry Score: A comprehensive, standardized system for quantitating renal tumor size, location, and depth. J Urol 2009;182:844-53. https://doi. org/10.1016/i.juro.2009.05.035

11. Imkamp F, Tolkach Y, Wolters $M$, et al. Initial experiences with the Hemopatch ${ }^{\circledR}$ as a hemostatic agent in zero-ischemia partial nephrectomy. World J Urol 2015;33:1527-34. https://doi.org/10.1007/ s00345-014-1404-4

12. Hupe $M C$, Büttner M, Tabrizi PF, et al. Hemopatch ${ }^{\circledast}$ as a hemostatic agent is safe in partial nephrectomy: A large, single-surgeon, retrospective evaluation. Adv Ther 2021;38:1024-34. https://doi.org/10.1007/ s12325-020-01584-8
13. Antonelli A, Minervini A, Mari A, et al. TriMatch comparison of the efficacy of FloSeal versus TachoSil vs. no hemostatic agents for partial nephrectomy: Results from a large, multicenter dataset. Int I Urol 2015;22:47-52. https://doi.org/10.1111/iju.12603

14. Shigeta K, Matsumoto K, Abe T, et al. The efficacy of the TachoSil binding suturing technique in laparoscopic partial nephrectomy to prevent the development of pseudoaneurysm. Asian I Surg 2020;43:668-75. https://doi.org/10.1016/i.asisur.2019.09.002

Correspondence: Dr. Ryan McLarty, Division of Urology, Department of Surgery, University of Alberta, Edmonton, AB, Canada; mclarty@ualberta.ca 